\title{
Pharmacokinetics and Relative Bioavailability of Ambroxol Hydrochloride Aerosol and Injection
}

\author{
Jing Zhang ${ }^{1}$, Na Yi ${ }^{1}$, Lidao Bao ${ }^{1,2}$, Tengfei Yu ${ }^{1, ~ * ~}$ \\ ${ }^{1}$ School of Pharmacy, Inner Mongolia Medical University, Hohhot, China \\ ${ }^{2}$ Department of Pharmacy, Affiliated Hospital of Inner Mongolia Medical University, Hohhot, China
}

Email address:

349881704@qq.com (Tengfei Yu)

\section{To cite this article:}

Jing Zhang, Na Yi, Lidao Bao, Tengfei Yu. Pharmacokinetics and Relative Bioavailability of Ambroxol Hydrochloride Aerosol and Injection. American Journal of Clinical and Experimental Medicine. Vol. 3, No. 6, 2015, pp. 368-371. doi: 10.11648/j.ajcem.20150306.18

\begin{abstract}
To compare the pharmacokinetics and relative bioavailability of ambroxol hydrochloride (AH) aerosol and injection, the volunteers were subjected to single-dose crossover inhalation (injection) of $100 \mathrm{mg}$ AH aerosol (injection). The drug concentrations in plasma were determined by HPLC. The areas under curve (AUC) of the two formulations were compared by the three-factor analysis of variance and the bi-directional one-side $t$ test. The $C_{\max }, t_{\max }$, $t 1 / 2 \beta$ and AUC values of the aerosol group and the injection group were $(154.75 \pm 26.12)$ and $(157.39 \pm 26.09) \mathrm{ng} / \mathrm{ml},(1.12 \pm 0.34)$ and $(1.29 \pm 0.33) \mathrm{h}$, $(6.98 \pm 1.62)$ and $(7.75 \pm 1.26) \mathrm{h}$, and $(1593.02 \pm 290.45)$ and $(1438 \pm 132.46) \mathrm{h} \cdot \mathrm{ng} / \mathrm{ml}$, respectively. The bioavailabilities of AH aerosol and injection were the same, and the relative bioavailability of the aerosol was $(96.52 \pm 11.44) \%$.
\end{abstract}

Keywords: Ambroxol Hydrochloride, Bioavailability, Pharmacokinetics, HPLC

\section{Introduction}

Ambroxol hydrochloride $(\mathrm{AH})$, which is also known as bromo-cyclohexylamine alcohol hydrochloride, is a new generation of phlegm dissolver that facilitates bronchial cilia evacuation and contributes to the clearance of airway secretions. Therefore, AH has been mainly used to treat acute and chronic respiratory diseases, especially to eliminate the phlegm of chronic bronchitis patients [1,2]. This study established a HPLC method to determine the AH concentrations in blood and investigated the pharmacokinetics of $\mathrm{AH}$ aerosol (made in China) and injection (imported) in healthy human body. The bioavailabilities of the two formulations were compared by the three-factor analysis of variance and the bi-directional one-side $\mathrm{t}$ test, which will provide evidence for clinical use [3].

\section{Materials and Methods}

\subsection{Reagents}

AH aerosol (trade name: Mucosolvan; approval number: $\mathrm{H} 20030360$ ) that was manufactured by Boehringer Ingelheim (Shanghai) pharmaceuticals Co., Ltd. was used for the control group, and $\mathrm{AH}$ injection $(300 \mathrm{mg} / 100 \mathrm{ml}$, approval number:
H19980178) that was produced by Jiangsu Hengrui Medicine Co., Ltd. was used for the trial group. AH reference substance (content: 99.6\%) was also provided by Jiangsu Hengrui Medicine Co., Ltd. Methanol, acetonitrile and tetrahydrofuran were of HPLC grade. Distilled water was used throughout the experiment. Ethyl ether, potassium dihydrogen phosphate, disodium hydrogen phosphate, hydrochloric acid, sodium hydroxide, potassium chloride, and phosphoric acid were of AR grade. The prepared buffer was filtered through a $0.50 \mu \mathrm{m}$ membrane. Preparation of $0.1 \mathrm{~mol} / \mathrm{L} \mathrm{pH} 9.0$ PBS buffer: 10.284 $\mathrm{g}$ potassium chloride was added to $8.528 \mathrm{~g}$ phosphoric acid, to which was added water till $1000 \mathrm{ml}$. Then $500 \mathrm{ml}$ of the resulting solution was taken out, to which were added $476 \mathrm{ml}$ of $0.1 \mathrm{~mol} / \mathrm{L} \mathrm{NaOH}$ and water until $1000 \mathrm{ml}$. Preparation of 0.1 mol/L pH 7.0 PBS buffer: $0.48 \mathrm{~g}$ potassium dihydrogen phosphate and $0.97 \mathrm{~g}$ disodium hydrogen phosphate were dissolved in $1000 \mathrm{ml}$ of water.

\subsection{Determination of AH Concentrations in Blood}

AH concentrations in blood were determined by HPLC.

\subsubsection{Apparatus and HPLC Conditions}

Hitachi 655 HPLC, including 655A-12 type pump, 655A22 type variable-wavelength UV detector and 655B-71 type microprocessor; Rheodyne 87125 type injection valve 
equipped with a $50 \mu \mathrm{l}$ quantitative tube; Resolve C18 HPLC column (Dalian Institute of chemical physics, Chinese Academy of Sciences, $4.6 \mathrm{mmx} 250 \mathrm{~mm}, 5 \mu \mathrm{m})$; mobile phase: acetonitrile: methanol: $0.1 \mathrm{~mol} / \mathrm{L} \mathrm{pH} 7.0$ phosphate buffer: tetrahydrofuran (300:300:200:20), flow rate: $2.5 \mathrm{ml} / \mathrm{min}$, sensitivity: 0.01 AUFs, detection wavelength: $242 \mathrm{~nm}$.

\subsubsection{Blood Sample Treatment}

$1.0 \mathrm{ml}$ of plasma was transferred into a $10 \mathrm{ml}$ plugged glass centrifuge tube, to which were added $0.5 \mathrm{ml}$ of $0.1 \mathrm{~mol} / \mathrm{L} \mathrm{pH}$ 9.0 PBS buffer and $5.0 \mathrm{ml}$ of ethyl ether. Then the sealed tube was volutedly mixed for $100 \mathrm{~s}$ and centrifuged at $3000 \mathrm{r} / \mathrm{min}$ for $6 \mathrm{~min}$. Then the ether layer was transferred to another 10 $\mathrm{ml}$ clean plugged glass centrifuge tube, to which was added $250 \mu \mathrm{l}$ of $0.1 \mathrm{~mol} / \mathrm{L} \mathrm{HCl}$. The resulting product was volutedly mixed for $100 \mathrm{~s}$ and centrifuged at $3000 \mathrm{r} / \mathrm{min}$ for $6 \mathrm{~min}$. Thereafter $35 \mu \mathrm{l}$ of the acid layer was injected after discarding the ether layer.

\subsubsection{Plasma Standard Curve and Detection Limit}

Experimental plasma samples at 10, 20, 40, 80, 160 and $320 \mathrm{ng} / \mathrm{ml}$ were prepared by adding AH standard solution into 6 healthy human plasma samples. Then the concentrations were linearly regressed by the peak heights recorded after injecting the samples. The results show that the detection of $\mathrm{AH}$ concentrations in plasma had good linearity from 20 to $320 \mathrm{ng} / \mathrm{ml}$. The regression equation was $\mathrm{H}=2.352+1.486 \mathrm{c} \quad(\mathrm{r}=0.998)$, the detection limit of $\mathrm{AH}$ in plasma was $4.5 \mathrm{ng} / \mathrm{ml}(\mathrm{S} / \mathrm{N}=2.8)$.

\subsubsection{Recovery and Precision}

AH plasma standard solutions at 20, 80, 160 and 320 $\mathrm{ng} / \mathrm{ml}$ were precisely prepared utilizing healthy human plasma samples. Then the solutions were injected according to the injection methods of blood samples. Absolute recoveries (ratio of peak height to the concentration of standard solution) and relative recoveries (ratio of the calculated concentration to the real one) of the 4 four solutions were calculated as $(83.98 \pm 2.15) \%,(82.77 \pm 1.74) \%$, $(83.16 \pm 2.27) \%$ and $(83.46 \pm 2.23) \%, \quad$ as well as $(100.9 \pm 6.68) \%, \quad(103.4 \pm 5.75) \%, \quad(102.7 \pm 4.84) \% \quad$ and $(99.7 \pm 5.29) \%$, respectively. AH plasma standard samples at $20,80,160$ and $320 \mathrm{ng} / \mathrm{ml}$ were precisely prepared, and the detections were repeated for 5 times within one day, yielding the intraday precision. The detections were continuously performed (once per day) for 7 days, yielding the day to day precision. The intraday and day to day RSD values of the samples at the 4 concentrations were $5.39 \%, 3.46 \%, 2.67 \%$ and $2.17 \%$, as well as $6.38 \%, 4.21 \%, 3.14 \%$ and $2.98 \%$, respectively.

\subsection{Selection of Volunteers and Sample Collection}

10 healthy male volunteers aging 18-32 years old (average: 19.46 \pm 6.91 ) and weighing 63-75 kg (average: $67.17 \pm 6.39$ ) were selected. All volunteers were confirmed normal by the routine examinations of liver, kidney and urine and electrocardiogram. They did not take any drugs 1 month before the trial, and they had signed consent forms. The trial had been approved by the Hospital Ethics Committee. The 10 volunteers were administered with $100 \mathrm{mg} \mathrm{AH}$ by inhalation and intravenous injection. Aerosol plus $5 \mathrm{ml}$ of saline were inhaled by an air compression pump. The inhalation each time lasted for 2-3 min, and the injection was given 4-6 ml $(10 \mathrm{mg} / 1 \mathrm{ml})$ per time, bid. The volunteers ate simultaneously $2.5 \mathrm{~h}$ after medication, the venous bloods $(5.0 \mathrm{ml})$ of which were collected before and $0.33,0.67,1.0,1.5,2,2.53,4,6,9$, 12,15 and $24 \mathrm{~h}$ after medication, respectively. Then the samples were centrifuged at $3000 \mathrm{r} / \mathrm{min}$ for $6 \mathrm{~min}$ after heparin anticoagulation, from which the plasma was separated. Thereafter the samples were stored at $-20^{\circ} \mathrm{C}$. Two trials were separated by 15 days.

\subsection{Calculation of Pharmacokinetics Parameters and Bioavailability}

The pharmacokinetics parameters were calculated automatically by the iteration fitting of program 3P87 utilizing the $\mathrm{AH}$ concentrations in blood determined at each time interval. AUC values were estimated by the ladder method using Mucosolvan as the reference to calculate the relative bioavailability of $\mathrm{AH}$ aerosol [4].

\subsection{Statistical Analysis}

The AUC values of the two administration methods were compared by the three-factor analysis of variance and the bidirectional one-side $t$ test utilizing SPSS 15.0, aiming to evaluate their bioavailabilities [5].

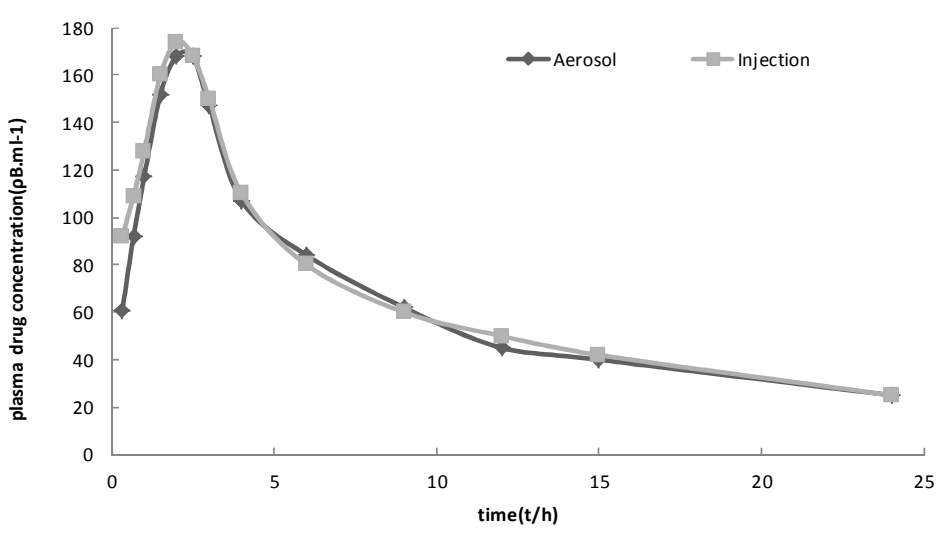

Figure 1. Dependences of the average AH concentrations in blood on time. 


\section{Results}

\subsection{AH Concentrations in Blood}

The volunteers were subjected to single-dose crossover inhalation (injection) of $100 \mathrm{mg} \mathrm{AH}$ aerosol (injection). The dependences of the average $\mathrm{AH}$ concentrations in blood on time are shown in Figure 1.

\subsection{Pharmacokinetics Parameters}

The automatic iteration fitting reveals that the pharmacokinetics of $\mathrm{AH}$ in healthy human body followed the two-compartment open model [6]. The relative parameters are listed in Table 1, and the AUC values are summarized in Table 2.

Table 1. Pharmacokinetics parameters $(n=10, x \pm s)$.

\begin{tabular}{|c|c|c|c|c|c|c|c|}
\hline Sample & $\mathrm{C}_{\max }\left(\mathrm{PB} /\right.$ ng.ml $\left.^{-1}\right)$ & $t_{\max }(t / h)$ & $\mathrm{t} 1 / 2 \mathrm{ka}(\mathrm{t} / \mathrm{h})$ & $t 1 / 2 \alpha(t / h)$ & $\mathbf{t} 1 / 2 \beta(t / h)$ & CL(L/h) & $\operatorname{AUC}\left(\mathrm{A} / \mathrm{h} \cdot \mathrm{ng} \cdot \mathrm{ml}^{-1}\right)$ \\
\hline Aerosol & $154.75 \pm 26.12$ & $1.12 \pm 0.34$ & $0.53 \pm 0.19$ & $1.46 \pm 1.10$ & $6.98 \pm 1.62$ & $215.67 \pm 101.7$ & $1593.02 \pm 290.45$ \\
\hline Injection & $157.39 \pm 26.09$ & $1.29 \pm 0.33$ & $0.71 \pm 0.16$ & $1.10 \pm 0.12$ & $7.75 \pm 1.26$ & $269.3 \pm 40.5$ & $1438 \pm 132.46$ \\
\hline
\end{tabular}

Table 2. AUC values.

\begin{tabular}{|c|c|c|c|c|c|}
\hline \multirow[b]{2}{*}{ No. } & \multicolumn{2}{|c|}{ Aerosol } & \multicolumn{2}{|c|}{ Injection } & \multirow{2}{*}{$\begin{array}{l}\text { F(\%) } \\
\text { AUC1/AUC2 }\end{array}$} \\
\hline & $\mathbf{P *}$ & $\operatorname{AUC1}\left(\mathrm{A} / \mathbf{h} \cdot \mathrm{ng} \cdot \mathrm{ml}^{-1}\right)$ & $P^{*}$ & AUC2 (A/h.ng.ml $\left.{ }^{-1}\right)$ & \\
\hline A & 2 & 1317.29 & 1 & 1510.21 & 87.23 \\
\hline B & 2 & 1402.77 & 1 & 1315.12 & 106.67 \\
\hline $\mathrm{C}$ & 1 & 1272.49 & 2 & 1237.65 & 102.82 \\
\hline $\mathrm{D}$ & 1 & 928.05 & 2 & 1219.34 & 76.11 \\
\hline $\mathrm{E}$ & 2 & 1564.95 & 1 & 1401.62 & 111.65 \\
\hline $\mathrm{F}$ & 1 & 1432.53 & 2 & 1529.61 & 93.65 \\
\hline G & 2 & 1641.51 & 1 & 1457.46 & 112.62 \\
\hline $\mathrm{H}$ & 1 & 1032.26 & 2 & 1108.21 & 93.15 \\
\hline I & 1 & 1263.28 & 2 & 1191.37 & 106.03 \\
\hline $\mathrm{J}$ & 2 & 879.96 & 1 & 1168.59 & 75.3 \\
\hline $\mathrm{x} \pm \mathrm{s}$ & \multicolumn{5}{|c|}{$96.523 \pm 11.44$} \\
\hline
\end{tabular}

\subsection{Statistical Analysis of Bioavailability}

The three-factor analysis of variance shows that the AUC values between different formulations, periods and individuals did not differ significantly $(\mathrm{P}>0.07)$. The bidirectional one-side $t$ test suggests that the bioavailabilities of $\mathrm{AH}$ aerosol and injection were identical $[7,8]$.

\section{Discussion}

This reserch to compare the pharmacokinetics and relative bioavailability of ambroxol hydrochloride $(\mathrm{AH})$ aerosol and injection, the volunteers were subjected to single-dose crossover inhalation (injection) of $100 \mathrm{mg} \mathrm{AH}$ aerosol (injection). The drug concentrations in plasma were determined by HPLC. The areas under curve (AUC) of the two formulations were compared by the three-factor analysis of variance and the bi-directional one-side $t$ test.

The experimental results of $\mathrm{AH}$ concentrations in blood indicate that $\mathrm{AH}$ was easily absorbable in both formulations. The pharmacokinetics parameters are consistent with those reported before. The $t 1 / 2 \beta$ and $c_{\max }$ of the two formulations did not differ significantly, whereas the $t_{\max }$ and $t 1 / 2 \mathrm{ka}$ of aerosol were apparently shorter than those of injection, suggesting aerosol was more easily absorbed [9].

The three-factor analysis of variance and the bi-directional one-side $t$ test of the AUC values of the two formulations did not differ significantly, indicating that both formulations could be similarly absorbed gastrointestinally and were of the identical bioavailability $((96.52 \pm 11.44) \%)$ [10].

\section{Conclusion}

Evaluation of hydrochloric acid ammonia bromine by the effectiveness of the aerosol inhalation medication, to hydrochloric acid ammonia bromine line by atomizing inhalation medication provides the theoretical basis of acute or chronic respiratory diseases.

\section{References}

[1] Hayashi K, Hosoe H, Kaise T, Ohmori K (2000) Protective effect of erdosteine against hypochlorous acid-induced acute lung injury and lipopolysaccharide-induced neutrophilic lung inflammation in mice. J Pharm Pharmacol 52(11): 1411-1416.

[2] Wiessmann KJ, Niemeyer K (1978) [Clinical results in the treatment of chronic obstructive bronchitis with ambroxol in comparison with bromhexine (author's transl)]. Arzneimittelforschung 28(5a): 918-921.

[3] Lee HJ, Joung SK, Kim YG, Yoo JY, Han SB (2004) Bioequivalence assessment of ambroxol tablet after a single oral dose administration to healthy male volunteers. Pharmacol Res 49(1): 93-98. 
[4] Ren YC, Wang L, He HB, Tang X (2009) Pulmonary selectivity and local pharmacokinetics of ambroxol hydrochloride dry powder inhalation in rat. J Pharm Sci 98(5): 1797-1803.

[5] Ballantyne BT, O'Hare SJ, Paschall JL, et al. (1993) Electromyographic activity of selected shoulder muscles in commonly used therapeutic exercises. Phys Ther 73(10): 668677; discussion 677-682.

[6] Amantea M, Newman MS, Sullivan TM, Forrest A, Working PK (1999) Relationship of dose intensity to the induction of palmar-plantar erythrodysesthia by pegylated liposomal doxorubicin in dogs. Hum Exp Toxicol 18(1): 17-26.

[7] Rutland BE, Nachreiner RF, Kruger JM (2009) Optimal testing for thyroid hormone concentration after treatment with methimazole in healthy and hyperthyroid cats. J Vet Intern Med 23(5): 1025-1030.

[8] Yuen O, Caligiuri MP, Williams R, Dickson RA (1996) Tardive dyskinesia and positive and negative symptoms of schizophrenia. A study using instrumental measures. Br J Psychiatry 168(6): 702-708.

[9] Cerretani D, Roviello F, Pieraccini M, et al. (2002) Pharmacokinetics of intraarterial mitomycin $\mathrm{C}$ in hypoxic hepatic infusion with embolization in the treatment of liver metastases. Vascul Pharmacol 39(1-2): 1-6.

[10] Villacampa J, Alcántar F, Rodríguez JM, Morales JM, Herrera J, Rosete R (2003) Pharmacokinetic properties of single-dose loratadine and ambroxol alone and combined in tablet formulations in healthy men. Clin Ther 25(8): 2225-2232. 